\title{
Characterization, pharmacokinetics, and hypoglycemic effect of berberine loaded solid lipid nanoparticles
}

This article was published in the following Dove Press journal:

International Journal of Nanomedicine

4 December 2013

Number of times this article has been viewed

\section{Mei Xue \\ Ming-xing Yang \\ Wei Zhang \\ Xiu-min Li \\ De-hong Gao \\ Zhi-min Ou \\ Zhi-peng Li \\ Su-huan Liu \\ Xue-jun Li \\ Shu-yu Yang}

Xiamen Diabetes Institute, The First Affiliated Hospital of Xiamen University, Xiamen, People's Republic of China
Correspondence: Shu-yu Yang

Xiamen Diabetes Institute, The First

Affiliated Hospital of Xiamen University,

55 Zhenhai Road, Xiamen, People's

Republic of China, 361003

$\mathrm{Tel}+865922137558$

Fax +86592 2137559

Email yang.shuyu@yahoo.com.cn

Xue-jun Li

Xiamen Diabetes Institute, The First

Affiliated Hospital of Xiamen University,

55 Zhenhai Road, Xiamen, People's

Republic of China, 361003

$\mathrm{Tel}+865922137610$

Fax +86592 2137558

Email lixuejun@yahoo.com.cn

\begin{abstract}
The high aqueous solubility, poor permeability, and absorption of berberine (BBR) result in its low plasma level after oral administration, which greatly limits its clinical application. BBR solid lipid nanoparticles (SLNs) were prepared to achieve improved bioavailability and prolonged effect. Developed SLNs showed homogeneous spherical shapes, small size (76.8 nm), zeta potential $(7.87 \mathrm{mV})$, encapsulation efficiency (58\%), and drug loading $(4.2 \%)$. The power of X-ray diffraction combined with ${ }^{1} \mathrm{H}$ nuclear magnetic resonance spectroscopy was employed to analyze chemical functional groups and the microstructure of BBR-SLNs, and indicated that the drug was wrapped in a lipid carrier. Single dose $(50 \mathrm{mg} / \mathrm{kg})$ oral pharmacokinetic studies in rats showed significant improvement $(P<0.05)$ in the peak plasma concentration, area under the curve, and variance of mean residence time of BBR-SLNs when compared to BBR alone $(P<0.05)$, suggesting improved bioavailability. Furthermore, oral administration of both BBR and BBR-SLNs significantly suppressed body weight gain, fasting blood glucose levels, and homeostasis assessment of insulin resistance, and ameliorated impaired glucose tolerance and insulin tolerance in $\mathrm{db} / \mathrm{db}$ diabetic mice. BBR-SLNs at high dose $(100 \mathrm{mg} / \mathrm{kg})$ showed more potent effects when compared to an equivalent dose of BBR. Morphologic analysis demonstrated that BBR-SLNs potentially promoted islet function and protected the islet from regeneration. In conclusion, our study demonstrates that by entrapping BBR into SLNs the absorption of BBR and its anti-diabetic action were effectively enhanced.
\end{abstract}

Keywords: berberine, solid lipid nanoparticles, pharmacokinetic, hypoglycemic effect

\section{Introduction}

Berberine (BBR), an isoquinoline alkaloid, is the major pharmacological component of the Chinese herb Coptis chinensis (Figure 1). BBR or BBR containing herbs have been used to treat intestinal infections, particularly bacterial diarrhea, for thousands of years in the People's Republic of China. ${ }^{1}$ Recently, a wealth of data has described the therapeutic effects of BBR on other diseases, such as congestive heart failure, cardiac arrhythmia, hypertension, hyperlipidemia, and cancer. ${ }^{2}$ In 1999 , Lee and Yuan reported the beneficial effects of BBR in treating diabetes. ${ }^{3,4}$ The proposed mechanisms of BBR's action include upregulation of the gene expression of hepatic low-density lipoprotein receptor, ${ }^{5}$ activation of adenosine monophosphate-activated protein kinase in adipose and muscle tissues, ${ }^{6}$ stimulation of glycolysis in peripheral tissues, ${ }^{7}$ promotion of insulin secretion, ${ }^{8}$ inhibition of liver gluconeogenesis, ${ }^{9}$ and promotion of intestinal glucagon-like protein-1 secretion. ${ }^{10}$ It was reported that the maximum concentration $\left(\mathrm{C}_{\max }\right)$ of $\mathrm{BBR}$ in plasma was $4 \mathrm{ng} / \mathrm{mL}$ after an oral administration of BBR at a dose of $100 \mathrm{mg} / \mathrm{kg}$ in rats, ${ }^{11}$ and only $0.4 \mathrm{ng} / \mathrm{mL}$ after a 


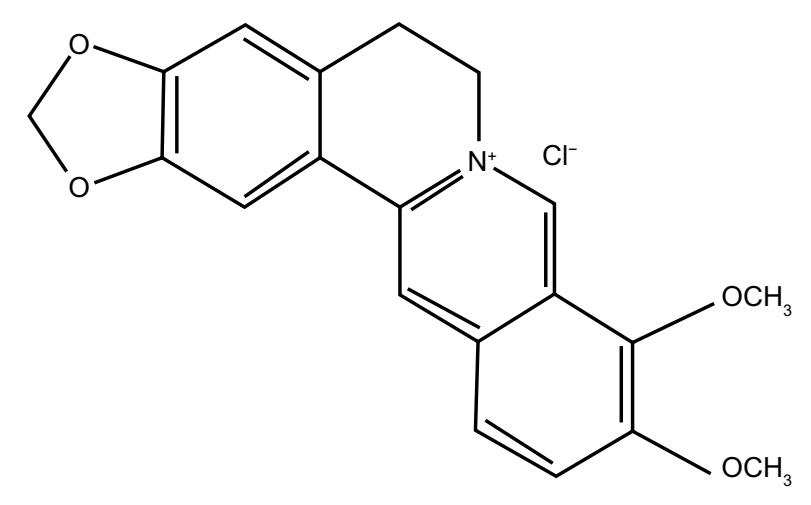

Figure I Structure of berberine.

single oral dose of $400 \mathrm{mg}$ BBR administration in humans, ${ }^{12}$ indicating a limited gastrointestinal BBR absorption. In addition, the bioavailability of BBR is poor $(<5 \%)^{13}$ and high dose (0.9-1.5 g/day in the clinic) administration causes gastrointestinal side effects, which greatly limit BBR's clinical applications. ${ }^{14}$ Therefore, it is extremely important to develop novel dosage forms of BBR to enhance its absorption and bioavailability, and thus facilitate its clinical applications.

Solid lipid nanoparticles (SLNs) have received great attention in recent years because of their various applications, including formulations for oral drug delivery. ${ }^{15}$ When compared to conventional formulations of bioactive compounds and plant extracts, SLN systems are reported to have more remarkable advantages, such as enhancing solubility and bioavailability, providing protection from toxicity, promoting pharmacological activity and stability, improving macrophage tissue distribution, maintaining sustained delivery, and protecting the drugs from physical and chemical degradation. ${ }^{15}$ Presently, the application of SLNs as carriers in facilitating the absorption of the active ingredient in traditional Chinese medicine, such as silymarin, triptolide, and paclitaxel, has been actively studied. ${ }^{16}$ We postulated that BBR loaded SLNs (BBR-SLNs) with an absorption enhancer may increase the absorption and bioavailability of BBR and attenuate its adverse effects. Therefore, the aim of the present study was to investigate the pharmacokinetic characteristics and the antidiabetic actions of BBR-SLNs, and thus provide new strategies in facilitating BBR's clinical applications.

\section{Materials and methods}

\section{Chemicals}

Reference grade BBR (purity quotient $>99.8 \%$ ), pluronic F-68, and mannitol were purchased from Sigma Chemical Co (St Louis, MO, USA). Soybean phospholipid and glycerol tripalmitate (purity quotient $>98 \%$ ) were obtained from Aladdin Industrial Corporation (Shanghai, People's Republic of China). Labrasol was supplied by Gattefosse (Nanterre Cedex, France). All other reagents were of high performance liquid chromatography (HPLC) grade or were of the highest purity commercially available.

\section{Animals}

All animal experiments were carried out in compliance with the standard ethical guidelines and were approved by Xiamen University Animal Care and Use Committee. Sixteen male Sprague Dawley (SD) rats, weighing 180-200 g, were purchased from Shanghai Experimental Animal Center, Chinese Academy of Sciences (Shanghai, People's Republic of China). Forty male db/db mice, weighing 30-40 g, were purchased from Experimental Animal Center of PLA Military Academy of Medical Sciences (Beijing, People's Republic of China). Animals were housed in an air conditioned room with a 12 hour/12 hour light/dark cycle and allowed to acclimate to the new environment for 1 week before the experiments.

\section{Preparations and characterization of BBR-SLNs}

\section{Preparations of BBR-SLNs}

BBR-SLNs were prepared using our patented method (No 201210495674.4, People's Republic of China patent). In brief, a mixture of lipid materials:glycerol tripalmitate:soybean phospholipid (1:6:6) was dissolved in ten volumes of mixed solution containing 5\% labrasol to form an organic phase. The mixed solution was composed of acetone and ethanol in equal proportions maintained at $80^{\circ} \mathrm{C}$, and was mixed until the dispersion appeared optically clear. The organic phase was injected slowly into an aqueous phase at the same temperature containing 1\% pluronic F-68 in double distilled water. A semitransparent pre-emulsion, appearing as a yellow transparent liquid, was obtained under constant magnetic agitation. After cooling to room temperature, the BBR-SLN suspensions were filtered through $0.45 \mu \mathrm{m}$ filters. Mannitol was added as a preservative agent, and the suspensions were then freeze dried.

\section{Particle size, zeta potential, and morphology}

The SLN formulation was characterized for particle size and zeta potential using dynamic light scattering with a Zetasizer 3000 HAS (Malvern Instruments Ltd., Malvern, UK). The microstructures of BBR-SLNs were examined by transmission electron microscopy ([TEM] H-7000, Hitachi, Tokyo, Japan) with a negative stain method. A drop of sample was 
applied to a copper grid coated with carbon film and air dried; $2 \%(\mathrm{w} / \mathrm{v})$ phosphotungstic acid solution was then dropped onto the grids for negative staining. After being air dried at room temperature, the samples were ready for TEM investigation.

\section{Drug loading (DL) and entrapment efficiency (EE)}

BBR-SLNs of the total drug content were determined by methanol emulsion breaking. The amount of drug that was not incorporated into the SLN was obtained by high speed centrifugation under low temperature $\left(30,000 \mathrm{rpm}, 4^{\circ} \mathrm{C}\right.$, 1 hour). The amount of BBR was determined by HPLC. Each experiment was performed in triplicate. EE and DL were calculated with the following formulae:

$$
\begin{gathered}
\mathrm{EE}(\%)=(\mathrm{C} 0-\mathrm{C}) / \mathrm{C} 0 \times 100 \% \\
\mathrm{DL}(\%)=(\mathrm{C} 0-\mathrm{C}) / \mathrm{C} 0 \times \mathrm{V} / \mathrm{M} \times 100 \%
\end{gathered}
$$

where $\mathrm{C}$ is the amount of drug encapsulated, $\mathrm{C} 0$ is the total amount of drug in the nanoparticle suspensions, $\mathrm{V}$ is final volume, and $\mathrm{M}$ is prescription in the amount of lipid.

\section{Powder X-ray diffraction (XRD) and nuclear magnetic resonance (NMR) measurements}

A combination of two spectroscopic methods was used to study the microstructure of BBR-SLNs. The BBR-SLNs lyophilized powder was uniformly spread on a glass substrate, and placed in a X-ray diffractometer for the diffraction experiments (Rigaku Corporation, Akishima, Tokyo, Japan). ${ }^{1} \mathrm{H}$ NMR spectra were recorded on a Varian NMR Spectrometer (500 MHz for 1H, Varian Medical Systems Inc, Palo Alto, CA, USA). A standard one dimensional pulse sequence PRESAT (presaturation) was used to obtain profiles of BBR with a 90 pulse length of about $10 \mu \mathrm{s}$ and spectra width of $20 \mathrm{ppm}$. A similarly processed blank SLN and free BBR sample were used as controls. Free BBR and blank lipid carrier were used as parallel controls.

\section{Pharmacokinetic studies}

\section{Study design}

Sixteen SD rats were randomly divided into two groups: BBR and BBR-SLNs (8 per group). The rats were fasted for 12 hours before the experiment and had free access to water. BBR $(50 \mathrm{mg} / \mathrm{kg})$ or BBR-SLNs $(50 \mathrm{mg} / \mathrm{kg})$ was administered via intragastric gavage. Blood samples were obtained before and $0.083,0.17,0.25,0.5,0.75,1,2,3,4,6,8,10$, and 12 hours after drug administration. The blood samples were centrifuged at $3,000 \mathrm{rpm}$ for 10 minutes at $4^{\circ} \mathrm{C}$. The plasma was collected and stored at $-20^{\circ} \mathrm{C}$.

\section{Sample preparation}

Forty microliters of tetrahydropalmatine $(1 \mu \mathrm{g} / \mathrm{mL})$ was added as internal standard (IS) to $0.2 \mathrm{~mL}$ of plasma. The samples were mixed, extracted with $10 \mathrm{~mL}$ acetonitrile by vortexing for 3 minutes, centrifuged at 3,500 rpm for 10 minutes, and $9 \mathrm{~mL}$ of the upper organic phase was carefully transferred to a $10 \mathrm{~mL}$ tube and evaporated with nitrogen. After drying, the residue was reconstituted in $200 \mu \mathrm{L}$ of $0.1 \%$ formic acid:acetonitrile $(50: 50, v / v)$, vortexed for 3 minutes and centrifuged at 15,000 rpm for 10 minutes at $4^{\circ} \mathrm{C}$. The supernatant $(50 \mu \mathrm{L})$ was collected and injected into the liquid chromatography-mass spectrometry-mass spectrometry (LC-MS-MS) system (Agilent 1260-6410, Agilent Technologies, Santa Clara, CA, USA).

\section{Chromatographic system and instrumentation for detection of the plasma concentration of BBR}

BBR was analyzed by LC-MS-MS comprising of a quaternary pump, an autoplate sampler, and a thermostatically controlled column compartment. The operation, acquisition, and analysis of data were controlled by Agilent Masshunter (Agilent Technologies). Chromatographic separations were performed with an Agilent Zorbax SB-C18 (2.1 mm, $50 \mathrm{~mm}, 1.8 \mu \mathrm{m})$ with a column temperature of $40^{\circ} \mathrm{C}$. The mobile phase was $0.1 \%$ formic acid (solvent $\mathrm{A}$ ) and acetonitrile (solvent $\mathrm{B}$ ). The gradient program used was: 0 min, solvent $A: B$ 75/25 (v/v); 2 minutes, solvent A:B 75/25; 3 minutes, solvent A:B 60/40; 5 minutes, solvent A:B 0/100; 8 minutes, A:B 0/100; 8.01 minutes, solvent $\mathrm{A}: \mathrm{B} 75 / 25$; and 12 minutes, solvent $\mathrm{A}: \mathrm{B} 75 / 25$. The flow rate was $0.3 \mathrm{~mL} / \mathrm{min}$. Electrospray ionization was used for mass analysis and detection. The mass spectrometer was operated in the positive ion detection mode. The ion source vaporization temperature was $350^{\circ} \mathrm{C}$, the drying gas flow was $10 \mathrm{~L} / \mathrm{min}$, and analysis was performed with selected reactions monitoring of the transitions with BBR: $[\mathrm{MH}+] \mathrm{m} / \mathrm{z} 336.1>321.0$; tetrahydropalmatine (IS): $[\mathrm{MH}+] \mathrm{m} / \mathrm{z} 356.2>192.2$.

\section{Data analysis}

Pharmacokinetic parameters, including the area under the plasma concentration versus time curve (AUC), volume of distribution, clearance, variance of mean residence time (VRT), and terminal elimination half-life $\left(t_{1 / 2}\right)$, were calculated by noncompartmental methods using DAS Software 2.0 (Chinese Pharmacological Society, Beijing, People's Republic of China). 


\section{Effects of BBR-SLNs on $\mathrm{db} / \mathrm{db}$ diabetic mice \\ Study design}

After one week of acclimatization, 40 male $\mathrm{db} / \mathrm{db}$ mice (9 weeks old) were randomly divided into four groups (10 per group): control group, BBR group (100 mg/kg), high dose BBR-SLNs (100 mg/kg), and low dose BBR-SLNs $(50 \mathrm{mg} / \mathrm{kg})$. Animals in the control group were treated with vehicle (saline) and the other groups were treated with respective drugs for 4 weeks via intragastric gavage. Body weight was monitored during the entire experiment, and food intake was measured after each mice was single caged for 3 days at the end of the experiment.

\section{Intraperitoneal glucose tolerance test (IPGTT), insulin tolerance test (IPITT), and serum insulin levels}

After overnight fasting, IPGTT was performed. Glucose (1 g/ $\mathrm{kg}$ body weight) was intraperitoneally injected and blood glucose was measured at time points of $0,15,30,60,90$, and 120 minutes using a blood glucose meter (LifeScan Inc, Milpitas, CA USA). The IPITT was conducted after 6 hours of food deprivation, followed by intraperitoneal injection of insulin (0.75 IU/kg body weight, Novo Nordisk, Denmark). Blood glucose was measured before and at 15, 30, 60, 90, and 120 minutes after insulin injection. The trapezoidal rule was used to calculate AUC (area under concentration-time curve) values for glucose.

At end of the experiment, after 12 hours of food deprivation, animals were anesthetized and sacrificed for tissue collection. Blood was collected from the orbital plexus and centrifuged at $3,000 \mathrm{rpm}$ at $4^{\circ} \mathrm{C}$ for 15 minutes. Enzymelinked immunosorbent assay kits (Mercodia AB, Uppsala, Sweden) were used to determine serum insulin levels following the manufacturer's instructions. Homeostasis assessment of insulin resistance (HOMA-IR) was calculated according to the formula:

$$
\begin{aligned}
\text { HOMA-IR }= & \text { Fasting insulin }(\mu \mathrm{U} / \mathrm{mL}) \\
& \times \text { Fasting glucose }(\mathrm{mmol} / \mathrm{L}) \\
& / 22.5 .
\end{aligned}
$$

\section{Histopathology}

The pancreas was harvested, weighed, and fixed in 10\% buffered formalin. Each pancreas was then processed for histological examination according to the conventional methods and stained with hematoxylin and eosin. The morphology of any lesion observed was classified and registered in an observerblind manner with respect to the treatment groups.

\section{Statistical analysis}

Data are expressed as mean \pm standard deviation. Statistical analysis was performed using one-way analysis of variance or Student's $t$-test when applicable. $P<0.05$ was accepted as statistically significant.

\section{Results}

\section{Characterization of BBR-SLN}

Particle size, zeta potential, DL, and EE

The mean particle size, polydispersity index, and zeta potential were $76.8 \mathrm{~nm}, 0.402$, and $7.87 \mathrm{mV}$, respectively (the PDI [polydispersity index] was well below 0.3 indicating a narrow particle size distribution). EE and DL were 58\% and $4.2 \%$, respectively.

\section{Particle shape and surface morphology}

The nanoparticles showed homogeneous spherical shapes under TEM, and the particle size ranged from 50 to $150 \mathrm{~nm}$ (original magnification $1 \times 10^{5}$ ) (Figure 2).

\section{Powder XRD and NMR measurements}

The XRD pattern (Figure 3) showed that the crystal diffraction peak of BBR disappeared completely in BBR-SLNs, indicating that the BBR based amorphous structure is dispersed in the nanoparticles. Partially amorphous nanosuspensions and the polymorphs were conductive to dissolution and absorption of the drug. Figure 4 shows ${ }^{1} \mathrm{H}$ NMR spectra of free BBR and BBR-SLNs. It is worth noting that ${ }^{1} \mathrm{H}$ NMR spectra of BBR-SLNs contains free BBR and lipid carrier. By combining the two spectroscopic methods, the microstructure

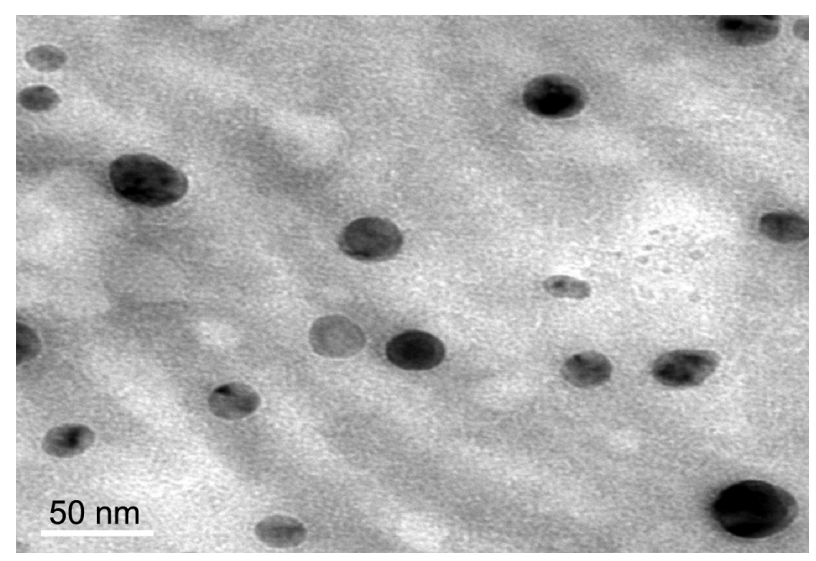

Figure 2 Microphotograph of berberine solid lipid nanoparticles. 


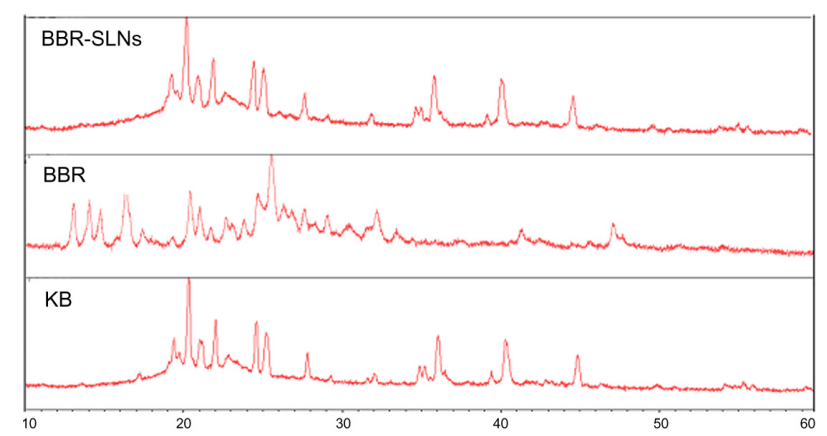

Figure 3 X-ray diffraction of berberine solid lipid nanoparticles.

Abbreviations: BBR, berberine; BBR-SLNs, berberine solid lipid nanoparticles; KB, blank nanoparticles without drug prepared with the same preparations of berberine solid lipid nanoparticles.

of BBR-SLNs was analyzed, and this initially demonstrated that the drug was wrapped in a lipid carrier.

\section{Pharmacokinetic studies} LC-MS-MS method validation

The retention times of BBR and tetrahydropalmatine were approximately 5.2 minutes and 2.9 minutes, respectively. The selected reactions monitoring chromatograms for control plasma, standard BBR, and IS in plasma after intragastric drug administration are shown in Figure 5. The calibration curves were linear over the range of $500 \mathrm{ng} / \mathrm{mL}$ and the limit of detection was $0.5 \mathrm{ng} / \mathrm{mL}$ ( signal to noise ratio $>10$ ). The correlation coefficient $\left(r^{2}\right)$ for plasma was 0.9994 . The methodology recoveries were greater than $80 \%$ and the intra-day and inter-day relative standard deviations were less than $10 \%$ at high, medium, and low concentrations. Plasma samples were stable in the following cases: 1) frozen and thawed three times, 2) stored for 12 hours at $4^{\circ} \mathrm{C}$, and 3 ) stored for 1 month at $-20^{\circ} \mathrm{C}$. The developed method was used successfully for determining BBR concentration in rat plasma and meets the requirements of biological sample analysis.

\section{Pharmacokinetics}

Pharmacokinetic parameters were calculated using DAS 2.0 software, according to the principle of determining the best correlation between the observed and computed concentrations. Whole parameters are shown in Table 1. The main pharmacokinetic parameters for BBR-SLNs were: $\mathrm{AUC}_{0-\mathrm{t}}, 113.57 \pm 72.93 \mu \mathrm{g} \cdot \mathrm{h} / \mathrm{L} ; \mathrm{C}_{\max }, 44.65 \pm 4.77 \mu \mathrm{g} / \mathrm{L}$; $\mathrm{t}_{1 / 2}, 11.50 \pm 10.78$ hours; and $\mathrm{VRT}_{0-\mathrm{t}}, 42.58 \pm 21.82$ hours. Figure 6 shows the plasma concentration time curves for BBR and BBR-SLNs after a single administration $(50 \mathrm{mg} / \mathrm{kg})$ to the rats. When compared with the BBR group, analysis of plasma from the BBR-SLNs group revealed a significant increase in $\mathrm{C}_{\max }(P<0.05)$, AUC $(P<0.05)$, and $\mathrm{VRT}_{0 \rightarrow \mathrm{t}}$ $(P<0.05)$, suggesting that nanoformulations can reduce fluctuations in drug concentrations, promote absorption, and possess a slow release character.

\section{Hypoglycemic effect of BBR-SLNs} Effects of BBR-SLNs on body weight and food intake When compared with the control group (vehicle), BBRSLNs $100 \mathrm{mg} / \mathrm{kg}$ treatment for 4 weeks suppressed the body weight gain of the mice, whereas animals treated with BBR $100 \mathrm{mg} / \mathrm{kg}$ or BBR-SLNs $50 \mathrm{mg} / \mathrm{kg}$ displayed no significant difference (Figure 7A). Food intake was not significantly different between vehicle and BBR or BBR-SLNs treated

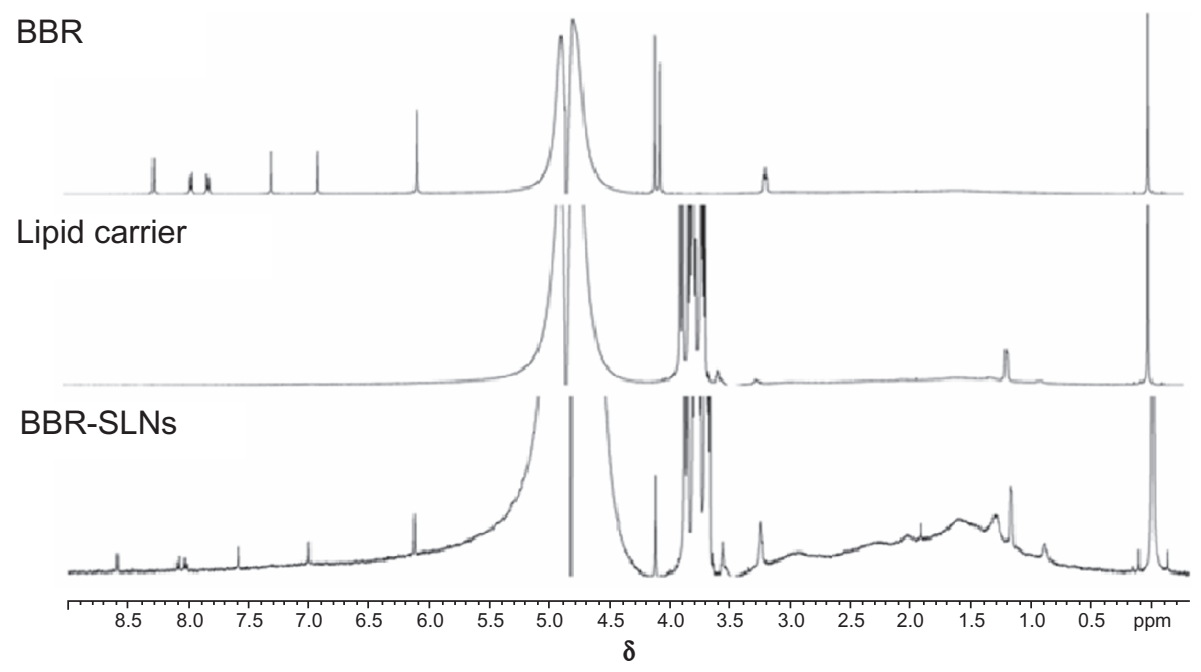

Figure 4 ' $\mathrm{H}$ nuclear magnetic resonance spectra of berberine solid lipid nanoparticles. Abbreviations: BBR, berberine; BBR-SLNs, berberine solid lipid nanoparticles. 

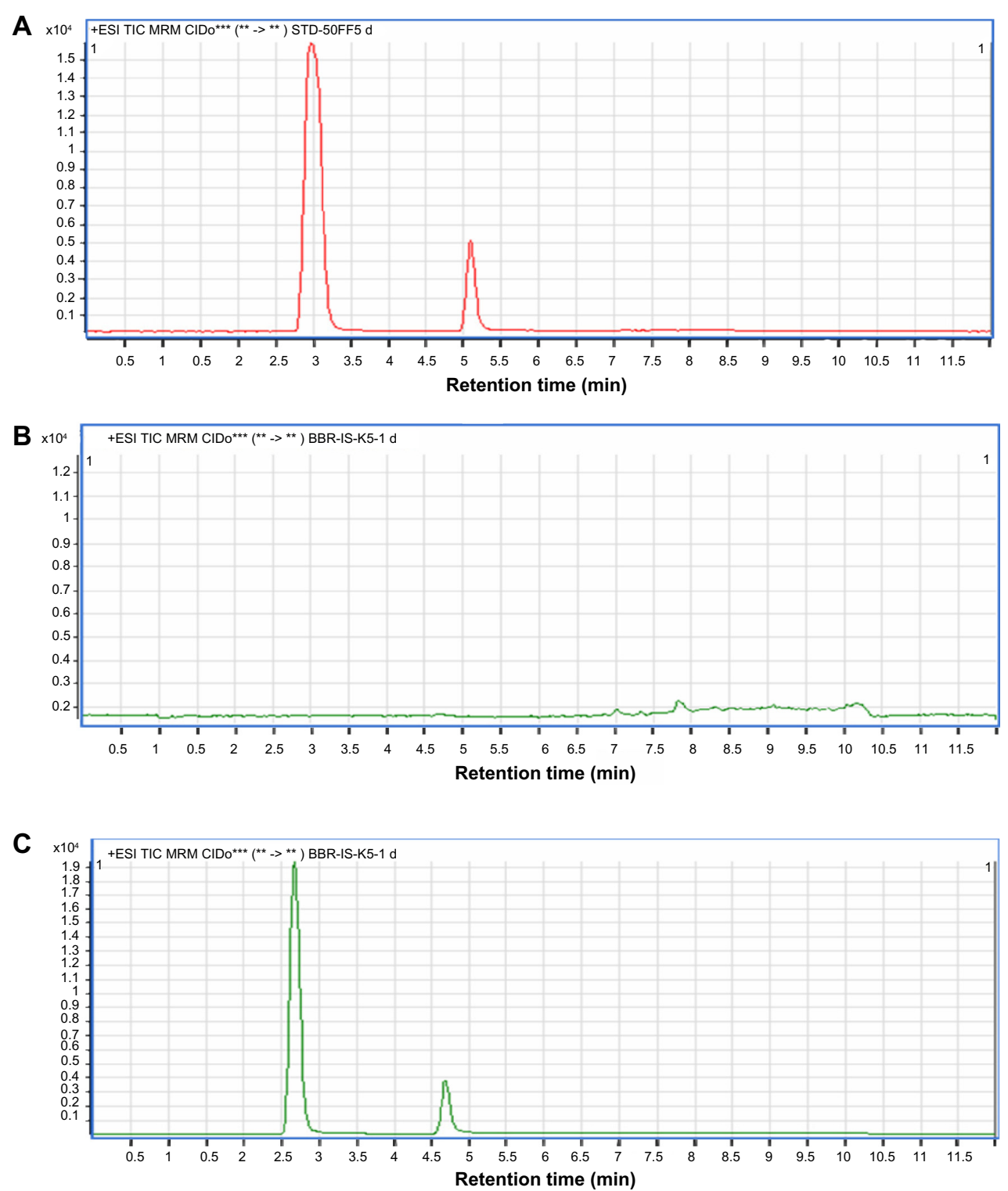

Figure 5 The selected reactions monitoring chromatograms for berberine.

Notes: (A) Standard berberine and internal standard in control plasma. (B) Blank plasma. (C) Plasma sample after intragastric administration.

groups (Figure 7B). Consistently, the expression of several hypothalamic neuropeptides known to be involved in regulating food intake was not significantly affected by BBR treatment.

\section{BBR-SLNs improved glucose tolerance and insulin sensitivity in $\mathrm{db} / \mathrm{db}$ mice}

When compared with vehicle, BBR-SLNs significantly decreased the fasting blood glucose level (Figure 8A) and elevated fasting serum insulin level in the $\mathrm{db} / \mathrm{db}$ mice (Figure 8B). The effects of BBR-SLNs at a dosage of $50 \mathrm{mg} / \mathrm{kg}$ were comparable with BBR at $100 \mathrm{mg} / \mathrm{kg}$, while BBR-SLNs at $100 \mathrm{mg} / \mathrm{kg}$ exerted more potent effects. Decreased HOMO-IR indices were seen in the BBR and
BBR-SLNs treated groups but not vehicle treated mice (Figure 8C). IPGTT showed that blood glucose levels in BBR treated mice were significantly lower at 30 and 60 minutes when compared to that in vehicle treated mice (Figure 8D), and the AUC in BBR-SLNs $100 \mathrm{mg} / \mathrm{kg}$ dose group was significantly decreased when compared to the BBR $100 \mathrm{mg} / \mathrm{kg}$ dose group (Figure $8 \mathrm{E}, P<0.05$ ). The IPITT results revealed that the blood glucose level was markedly lower in BBR and BBR-SLNs treated mice when compared to vehicle treated mice (Figure 8F), suggesting remarkably improved insulin sensitivity. There was a more significant reduction in the AUC of BBR-SLNs $100 \mathrm{mg} / \mathrm{kg}$ group than in the BBR $100 \mathrm{mg} / \mathrm{kg}$ group (Figure 8G, $P<0.05$ ). Conclusively, these data revealed that BBR can improve insulin sensitivity in 
Table I Pharmacokinetic parameters of berberine after a single intragastric administration of $50 \mathrm{mg} / \mathrm{kg}$ to rats (mean \pm standard deviation, $\mathrm{n}=7$ )

\begin{tabular}{|c|c|c|c|}
\hline Parameters & Unit & BBR & BBR-SLNs \\
\hline$A \cup C_{(0-t)}$ & $\mu g / L \cdot h$ & $56.478 \pm 29.6 I$ & $113.57 \pm 72.927^{*}$ \\
\hline $\mathrm{AUC}_{(0-\infty)}$ & $\mu g / L \cdot h$ & $86.417 \pm 40.84$ & $179.384 \pm 160.07$ \\
\hline $\mathrm{AUMC}_{(0-\mathrm{t})}$ & & $479.425 \pm 425.34$ & $\mathrm{I}, 030.96 \pm 88 \mathrm{I} .54$ \\
\hline $\operatorname{AUMC}_{(0-\infty)}$ & & $\mathrm{I}, 390.635 \pm 1,156.94$ & $4,648.305 \pm 634.55$ \\
\hline $\mathrm{MRT}_{(0-\mathrm{t})}$ & $\mathrm{H}$ & $7.647 \pm 2.68$ & $8.222 \pm 2.43$ \\
\hline $\mathrm{MRT}_{(0-\infty)}$ & $\mathrm{H}$ & $15.159 \pm 6.65$ & $18.307 \pm 12.80$ \\
\hline $\mathrm{VRT}_{(0-\mathrm{t})}$ & $h^{\wedge} 2$ & $23.398 \pm 13.92$ & $42.577 \pm 21.82^{*}$ \\
\hline $\operatorname{VRT}_{(0-\infty)}$ & $h^{\wedge} 2$ & $216.949 \pm 20.11$ & $500.025 \pm 72.66$ \\
\hline$t_{1 / 2} z$ & $\mathrm{H}$ & $9.228 \pm 5.13$ & $11.504 \pm 10.78$ \\
\hline $\mathrm{T}_{\max }$ & $\mathrm{H}$ & $2.25 \pm 3.68$ & $0.38 I \pm 0.7 \mid$ \\
\hline $\mathrm{CLz} / \mathrm{F}$ & $\mathrm{L} / \mathrm{h} / \mathrm{kg}$ & $685.365 \pm 290.96$ & $5|8.523 \pm 365.4|$ \\
\hline $\mathrm{Vz} / \mathrm{F}$ & $\mathrm{L} / \mathrm{kg}$ & $8,901.123 \pm 6,158.60$ & $6,873.35 \pm 887.02$ \\
\hline Zeta & & $0.115 \pm 0.10$ & $0.189 \pm 0.23$ \\
\hline $\mathrm{Cz}$ & $\mu g / L$ & $3.318 \pm 3.61$ & $2.657 \pm 3.47$ \\
\hline $\mathrm{C}_{\max }$ & $\mu g / L$ & $11.057 \pm 6.24$ & $44.651 \pm 4.77^{*}$ \\
\hline
\end{tabular}

Note: *Denotes $P<0.05$ in comparison with the berberine group.

Abbreviations: AUC, area under the curve; AUMC, area under the first-moment curve; $B B R$, berberine; BBR-SLN, berberine solid lipid nanoparticles; MRT, mean residence time; $\mathrm{Cz}$, concentration; $\mathrm{CLz}$, clearance rate; $\mathrm{C}_{\text {max }}$, peak plasma concentration; $\mathrm{F}, \mathrm{VRT}$, variance of mean residence time; $T_{\text {max }}$, time to peak plasma concentration; $t_{1 / 2}$, terminal elimination half-life; $\mathrm{Vz}$, volume.

$\mathrm{db} / \mathrm{db}$ diabetic mice, and the BBR-SLNs exerted more potent effects than an equivalent dose of BBR, probably through increased absorption and/or bioavailability.

\section{Histopathology}

Pancreas acinar of the BBR group, and BBR-SLNs at $100 \mathrm{mg} / \mathrm{kg}$ and $50 \mathrm{mg} / \mathrm{kg}$ dosages was of no difference, with intact islet cell structure and no obvious inflammatory response in the surrounding tissue (Figures 9 and 10). However, it can be seen that the number of islets in the treatment group was significantly more than the control group. The largest number of islets was in the BBR-SLNs high dosage group. But, there was no discernible difference between the BBR and BBR-SLNs $100 \mathrm{mg} / \mathrm{kg}$ groups.

\section{Discussion}

\section{Characterization of SLN}

More than $90 \%$ of the BBR-SLNs particles showed a small particle size $(76.8 \mathrm{~nm})$. SLNs were prepared using a solvent evaporation method which results in small particles. Small particle size less than $200 \mathrm{~nm}$ is desired for being usually invisible to the reticulo-endothelial system and for circulating over a prolonged period of time in vivo. Moreover, a zeta potential of $\geq \pm 25 \mathrm{mV}$ is recommended for achieving stable dispersions. This is attributed to the existence of repulsive forces between the particles, preventing them from contacting each other and agglomerating. Neutral particles obtained presently can thus be considered intrinsically stable as no interparticulate molecular interactions (both attractive and repulsive) are expected from these particles. In contrast to the neutral and negatively charged particles, the positively charged nanoparticles are taken up more rapidly by the cell membranes. ${ }^{17}$ The shape and the surface morphology of our prepared SLNs was studied by TEM. The TEM images confirmed that the particles were spherical in nature. The size

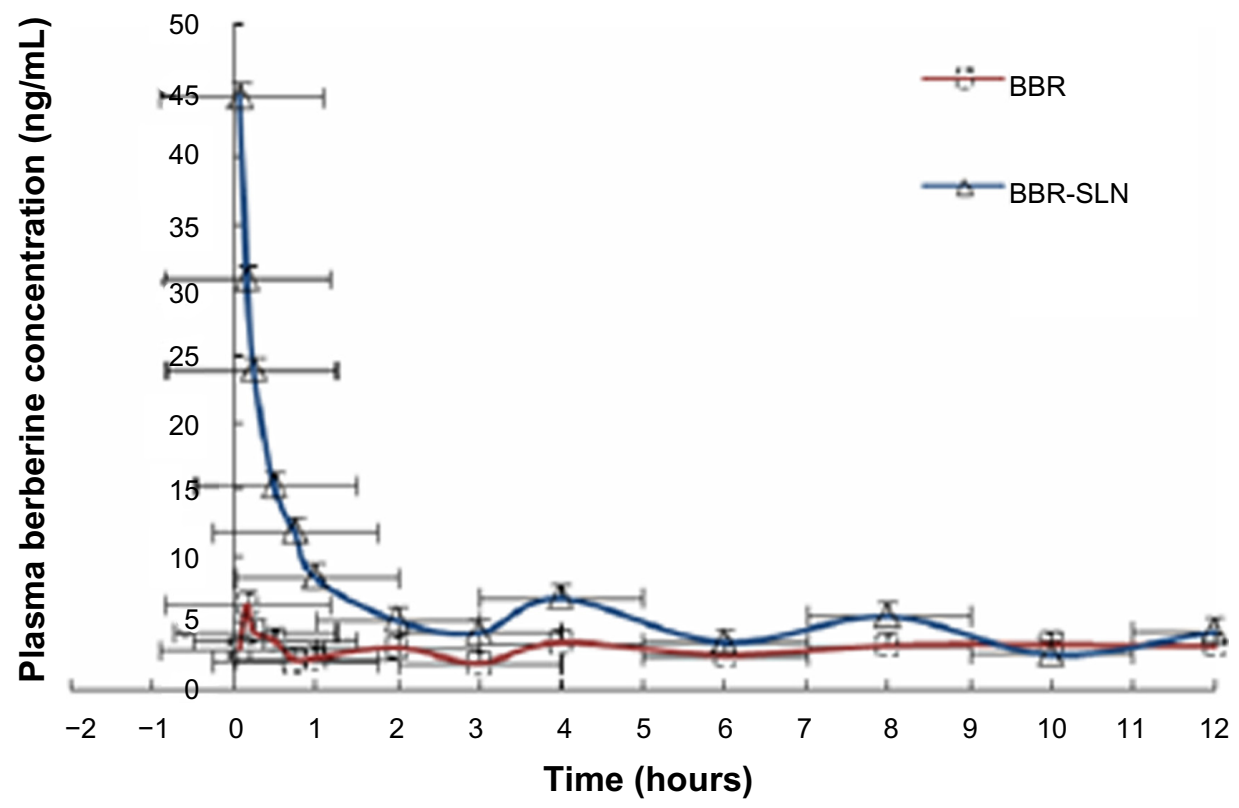

Figure 6 Plasma concentration time curve of berberine and berberine solid lipid nanoparticles after a single intragastric administration of $50 \mathrm{mg} / \mathrm{kg}$ to male rats. Abbreviations: BBR, berberine; BBR-SLNs, berberine solid lipid nanoparticles. 
A

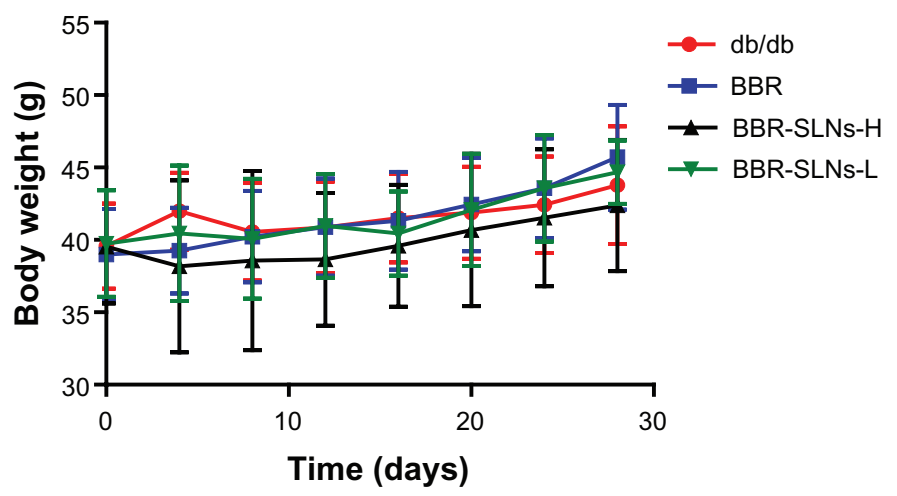

B

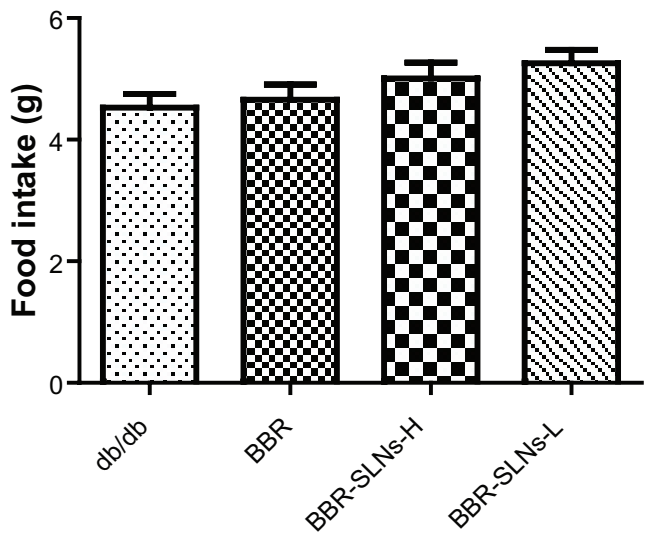

Figure 7 Effect of berberine and berberine solid lipid nanoparticles on body weight and food intake.

Notes: (A) Changes in body weight after a single ig administration $50 \mathrm{mg} / \mathrm{kg}$ to $\mathrm{db} / \mathrm{db}$ mice. (B) Changes in food intake after each rat was single caged for $3 \mathrm{days}$. Abbreviations: BBR, berberine; BBR-SLNs-H, berberine solid lipid nanoparticles high-dose group; BBR-SLNs-L, berberine solid lipid nanoparticles low-dose group; ig, intragastric.

and shape has been demonstrated to directly influence the uptake of nanoparticles into cells. ${ }^{19}$ The size dependent uptake of nanoparticles is likely related to the so-called membrane wrapping process, which governs how a membrane encloses a particle. It has been demonstrated that optimal endocytosis occurs when there is no ligand shortage on the nanoparticle and no localized receptor shortage on the cell surface. ${ }^{18-20}$ High recovery is a prerequisite for developing a manufacturing process of any formulation as it accounts for losses during the production process. The DL values of above $4.2 \%$ confirm suitability of the developed process and its applicability for scaling up. The EEs obtained (close to 59\%), to the best of our knowledge, have never been reported before for BBR or similar hydrophilic drugs. Polymorph is also an important factor affecting drug stability, solubility, dispersion, and pharmacodynamic actions. The power of XRD combined with ${ }^{1} \mathrm{H}$ NMR spectroscopy was employed to analyze the chemical functional groups and the microstructure of BBRSLNs, and proved that the drug was successfully wrapped in a lipid carrier. ${ }^{21}$ In addition, BBR-SLNs lyophilized powder was reconstituted after 6 months without changes in average particle size and zeta potential, indicating that the BBR-SLN was stable and can be stored over long periods of time.

\section{Pharmacokinetics}

In the pharmacokinetic studies, we first developed the current method of determining BBR concentration in rat plasma using LC-MS-MS. The validation of the bioanalytical method used for extraction of BBR from plasma, its recovery from spiked plasma samples, plasma sample stability, and limit of detection/limit of quantification were determined.
The developed method was more rapid and sensitive for determination of BBR in rat plasma.

After oral administration, nanoparticles can undergo direct gastrointestinal transit or adhere to the mucosa before elimination in feces. However, oral absorption can also occur, and nanoparticles are likely to cross the gastrointestinal barrier to deliver the drug content to the blood, lymph, and target organs. Direct contact and adhesion of nanoparticles to the mucosal surface is a prerequisite for particle degradation. With SLNs, the peak plasma concentration of BBR in rats was elevated, the peak time was delayed, and the AUC was increased, indicating an improved bioavailability of BBR. The pharmacokinetics of several orally administered drugs has been improved by means of nanoparticles, and there is evidence showing that lipid nanodispersions containing drugs maintained increased bioavailability and prolonged plasma levels after administration in animals. ${ }^{22-24}$ Our results are consistent with these reports, suggesting that the changes in pharmacokinetics may be an important mechanism underlying the enhanced efficacy on nanoformulations.

\section{Hypoglycemic effect of BBR-SLN}

In this study we have found that BBR-SLNs increase plasma BBR concentration and bioavailability in pharmacokinetic studies. Here we further investigated the antidiabetic effects of BBR-SLNs in vivo. A dose of BBR at $100 \mathrm{mg} / \mathrm{kg}$, based on the previous clinical and animal studies (0.9-1.5 g/days), has been applied to the $\mathrm{db} / \mathrm{db}$ mice. ${ }^{25}$ In agreement with a previous study, BBR actions were confirmed here in decreasing body weight, fasting blood glucose levels, HOMA-IR, and improving impaired glucose tolerance and insulin tolerance 
A

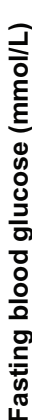

C

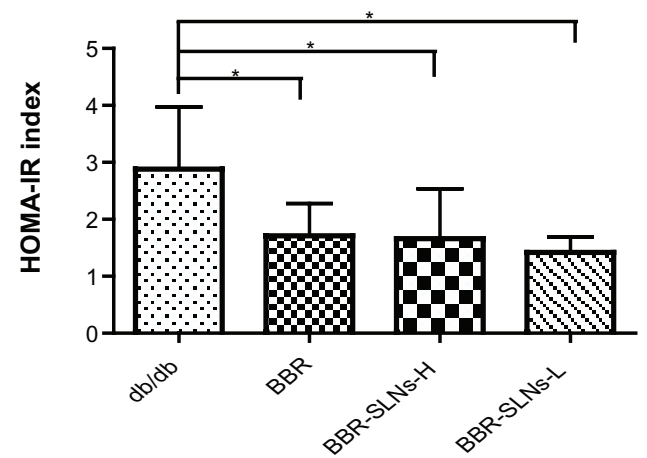

E
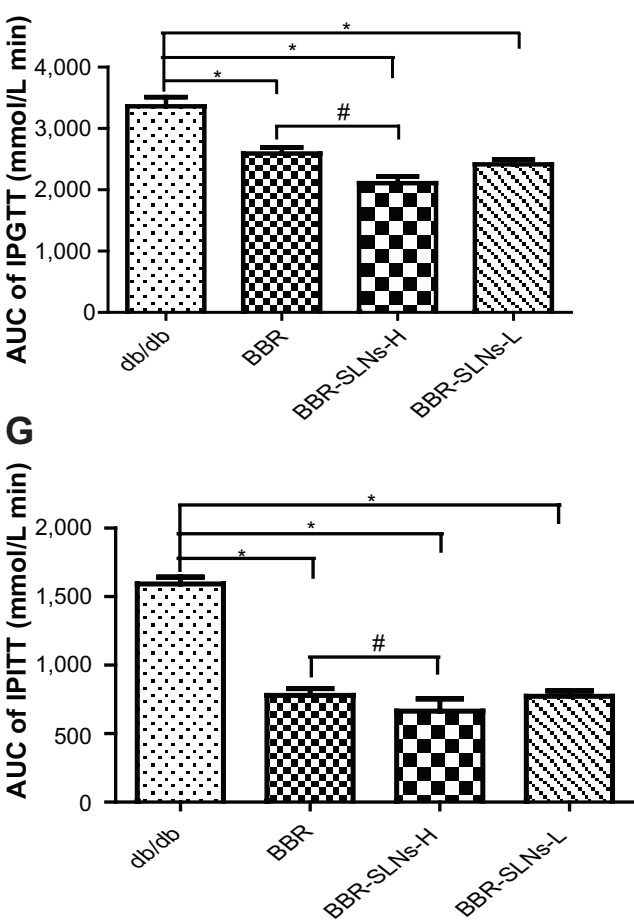

B

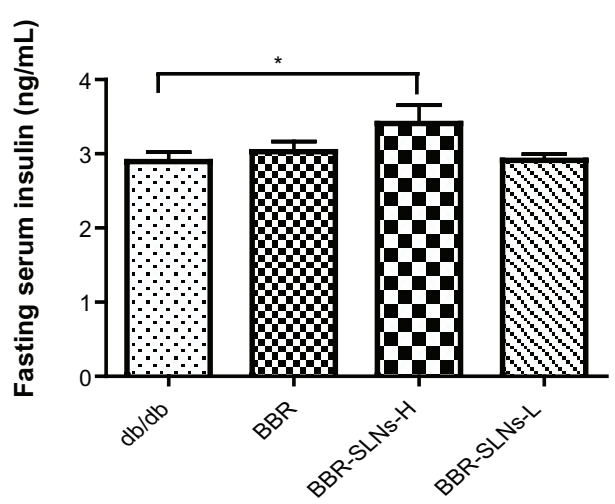

D

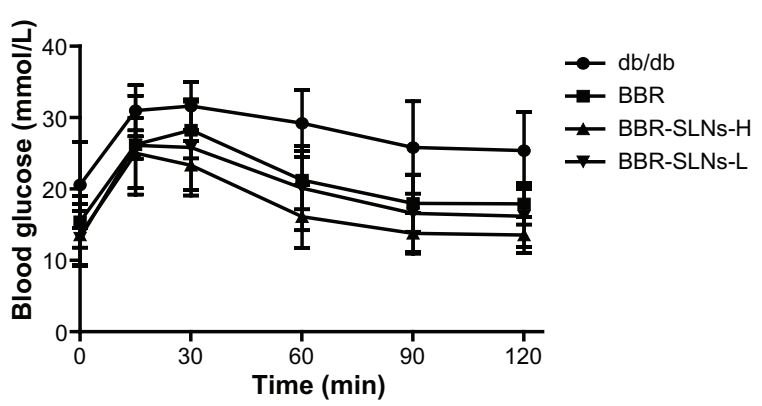

$\mathbf{F}$

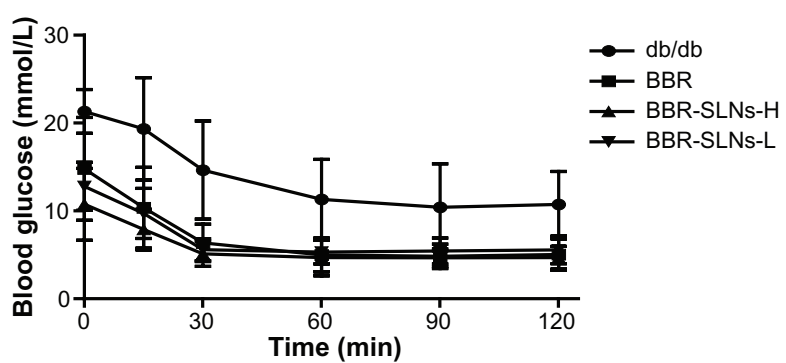

Figure 8 Preventive effects of berberine and berberine solid lipid nanoparticles on the development of insulin resistance in $\mathrm{db} / \mathrm{db}$ mice.

Notes: (A) Fasting blood glucose (FBG). (B) Fasting serum insulin (FINS). (C) Homeostasis assessment of insulin resistance. (D) Intraperitoneal glucose tolerance test. (E) Area under the curve of the intraperitoneal glucose tolerance test. (F) Insulin tolerance test. (G) Area under the curve of the insulin tolerance test. *Denotes $P<0.05$ versus the $\mathrm{db} / \mathrm{db}$ model group and \#denotes $P<0.05$ versus the berberine group.

Abbreviations: BBR, berberine; BBR-SLNs-H, berberine solid lipid nanoparticles high-dose group; BBR-SLNs-L, berberine solid lipid nanoparticles low-dose group; HOMAIR, homeostasis assessment of insulin resistance; AUC of IPGTT, area under the curve of the intraperitoneal glucose tolerance test; AUC of IPITT, area under the curve of insulin tolerance test. 
A

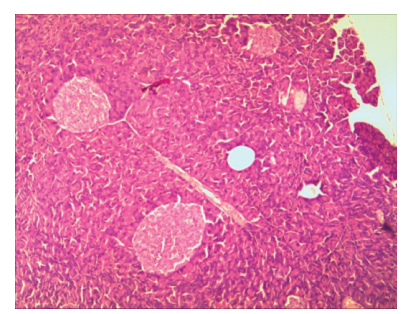

B

C

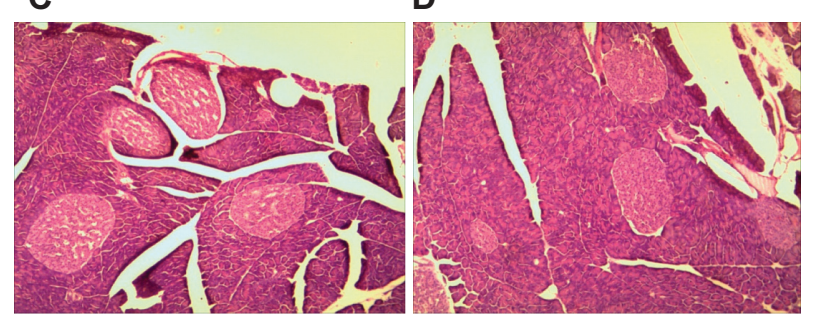

Figure 9 Histological changes in pancreas from rats of different treatment groups. Notes: (A) db/db model group. (B) Berberine group (100 mg/kg). (C) Berberine solid lipid nanoparticles group (100 mg/kg). (D) Berberine solid lipid nanoparticles group $(50 \mathrm{mg} / \mathrm{kg})$. Hematoxylin and eosin staining $(200 \times)$.

in diabetic mice. ${ }^{26} \mathrm{BBR}-\mathrm{SLNs}$ at $100 \mathrm{mg} / \mathrm{kg}$ dosage showed more potent effects than the equivalent dose of BBR in $\mathrm{db} / \mathrm{db}$ mice, especially in improving glucose tolerance and insulin sensitivity. In addition, BBR-SLNs increased the pancreatic islet numbers. Strategies to improve the absorption and efficacy of BBR have been investigated in other studies such as the combination with sodium caprate. ${ }^{27,28}$ The abnormal immune status in $\mathrm{db} / \mathrm{db}$ mice, which directly leads to the lack of islet function, may be systemically improved by BBR, thus facilitating islet regeneration, while the independent effect of lipid nanoparticles in regulating insulin and other lipoproteins should also be considered. Additionally, the carrier materials,

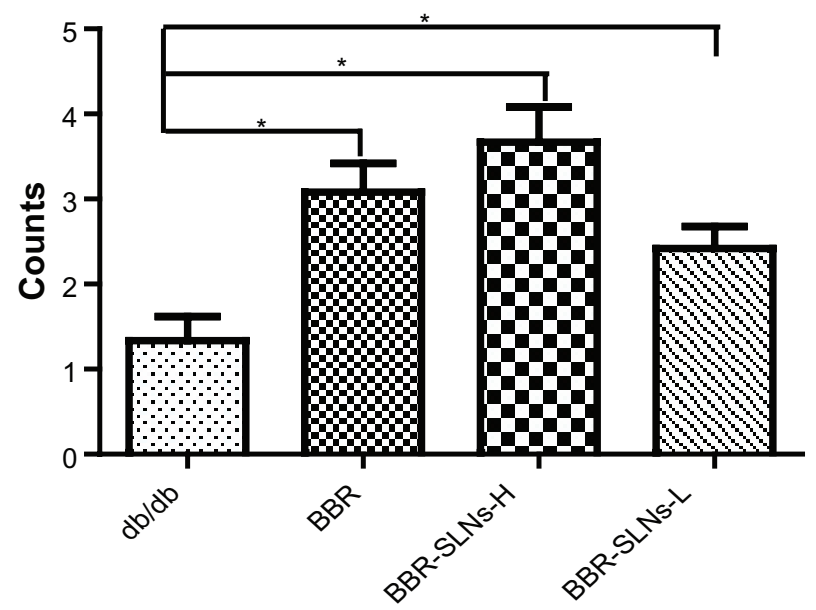

Figure 10 Changes in islet volume from histology with different perspectives (10 $\times 20$ magnification).

Note: *Denotes $P<0.05$ versus the $\mathrm{db} / \mathrm{db}$ control group.

Abbreviations: BBR, berberine; BBR-SLNs-H, berberine solid lipid nanoparticles high-dose group; BBR-SLNs-L, berberine solid lipid nanoparticles low-dose group. mainly including lecithin and tripalmitin, did not show toxic side effects in the experimental or pathological examination. Further studies investigating the hypoglycemic mechanisms of BBR-SLNs, toxicology, and other pharmacological effects are underway.

\section{Conclusion}

Our study on the characterization, pharmacokinetics, and hypoglycemic effect of BBR-SLNs demonstrated that the preparation method of BBR-SLNs is feasible with good quality and stability characteristics. SLNs can entrap BBR, which results in improved bioavailability of BBR in plasma. A SLN delivery system can enhance the antidiabetic action of BBR, and BBR-SLNs offer a promising delivery system to facilitate BBR antidiabetic clinical therapy and other applications.

\section{Disclosure}

The authors declare that they have no competing interests in this work.

\section{References}

1. Tang J, Feng Y, Tsao S, Curtain R, Wang N. Berberine and Coptidis rhizoma as novel antineoplastic agents: a review of traditional use and biomedical investigations. J Ethnopharmacol. 2009;126:5-17.

2. Yang J, Lin J. Advance on study in anti-tumor mechanism of berberine (Ber). Zhongguo Zhong Yao Za Zhi. 2007;32:881-883. Chinese.

3. Lee KZ. Clinical trials of berberine chloride as pharmaceutical agent of type II diabetes. Hubei J Tradit Chin Med. 2006;28:38-41.

4. Yuan YH. Clinical trial of berberine as pharmaceutical agent of type 2 diabetes. Mod J Integr Tradit Chin West Med. 1999;8:1777-1781.

5. Kong W, Wei J, Abidi P, et al. Berberine is a novel cholesterol-lowering drug working through a unique mechanism distinct from statins. Nat Med. 2004;10:1344-1351.

6. Lee YS, Kim WS, Kim KH, et al. Berberine, a natural plant product, activates AMP-activated protein kinase with beneficial metabolic effects in diabetic and insulin-resistant states. Diabetes. 2006;55: 2256-2264.

7. Yin J, Gao Z, Liu D, Liu Z, Ye J. Berberine improves glucose metabolism through induction of glycolysis. Am J Physiol Endocrinol Metab. 2008;294:E148-E156.

8. Ko B-S, Choi SB, Park SK, Jang JS, Kim YE, Park S. Insulin sensitizing and insulinotropic action of berberine from Cortidis rhizoma. Biol Pharm Bull. 2005;28:1431-1437.

9. Xia X, Yan J, Shen Y, et al. Berberine improves glucose metabolism in diabetic rats by inhibition of hepatic gluconeogenesis. PLoS One. 2011;6(2):e16556.

10. Lu SS, Yu YL, Zhu HJ, et al. Berberine promotes glucagon-like peptide-1 (7-36) amide secretion in streptozotocin-induced diabetic rats. J Endocrinol. 2009;200:159-165.

11. Liu Y, Hao H, Xie H, Lv H, Liu C, Wang G. Oxidative demethylenation and subsequent glucuronidation are the major metabolic pathways of berberine in rats. J Pharm Sci. 2009;98:4391-4401.

12. Hua W, Ding L, Chen Y, Gong B, He J, Xu G. Determination of berberine in human plasma by liquid chromatography-electrospray ionization-mass spectrometry. J Pharm Biomed Anal. 2007;44:931-937.

13. Qu QM, Zhang MF. The pharmacokinetics of berberine. Chin Acad Med Mag Organ. 2002;12:31-35. 
14. Liu YT, Hao HP, Xie HG, et al. Extensive intestinal first-pass elimination and predominant hepatic of berberine explain its low plasma levels in rats. Drug Metab Dispos. 2010;38:1779-1784.

15. Paolino D, Cosco D, Cilurzo F, Fresta M. Innovative drug delivery systems for the administration of natural compounds. Curr Bioact Compd. 2007;3:262-277.

16. Ajazuddin, Saraf S. Applications of novel drug delivery system for herbal formulations. Fitoterapia. 2010;81:680-689.

17. Lockman PR, Oyewumi MO, Koziara JM, Roder KE, Mumper RJ, Allen DD. Brain uptake of thiamine-coated nanoparticles. J Control Release. 2003;93:271-282.

18. Gratton SE, Ropp PA, Pohlhaus PD, et al. The effect of particle design on cellular internalization pathways. Proc Natl Acad Sci USA. 2008;105:11613-11618.

19. Albanese A, Tang PS, Chan WC. The effect of nanoparticle size, shape, and surface chemistry on biological systems. Annu Rev Biomed Eng. 2012;14:1-16.

20. Lu F, Wu SH, Hung Y, Mou CY. Size effect on cell uptake in wellsuspended, uniform mesoporous silica nanoparticles. Small. 2009;5: 1408-1413.

21. Chen YX. Nanomedicine Evaluation and Methods. Chemical Industry Press, Beijing: 2012:250-256.
22. Rohit B, Indu PK. Pharmacokinetics, tissue distribution and relative bioavailability of isoniazid-solid lipid nanoparticles. Int J Pharm. 2013;441(1-2):202-212.

23. Li BX, Yang BF, Hao XM, Zhou H, Sun MZ. Study on the pharmacokinetics of berberine in single dosage and coadministration with oryzanol in rabbits and healthy volunteers. Chin Pharm J. 2000;35: 33-35.

24. Liu Y, Hao H, Xie H, Lv H, Liu C, Wang G. Oxidative demethylenation and subsequent glucuronidation are the major metabolic pathways of berberine in rats. J Pharm Sci. 2009;98:4391-4401.

25. Lee YS, Kim WS, Kim KH, et al. Berberine, a natural plant product, activates AMP-activated protein kinase with beneficial metabolic effects in diabetic and insulin-resistant states. Diabetes. 2006;55:2256-2264.

26. Zhang X, Zhao YF, Zhang MH, et al. Structural changes of gut microbiota during berberine-mediated prevention of obesity and insulin resistance in high-fat rats. PLoS One. 2012;7:e42529.

27. Lv XY, Li J, Zhang M, et al. Enhancement of sodium caprate on intestine absorption and antidiabetic action of berberine. AAPS Pharm Sci Tech. 2010;11:372-382.

28. Kheir MM, Wang YG, Hua L, et al. Acute toxicity of berberine and its correlation with the blood concentration in mice. Food Chem Toxicol. 2010;48:1105-1110.
International Journal of Nanomedicine

\section{Publish your work in this journal}

The International Journal of Nanomedicine is an international, peerreviewed journal focusing on the application of nanotechnology in diagnostics, therapeutics, and drug delivery systems throughou the biomedical field. This journal is indexed on PubMed Central,

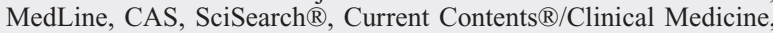

\section{Dovepress}

Journal Citation Reports/Science Edition, EMBase, Scopus and the Elsevier Bibliographic databases. The manuscript management system is completely online and includes a very quick and fair peer-review system, which is all easy to use. Visit http://www.dovepress.com/ testimonials.php to read real quotes from published authors. 